\title{
Diagnostic Value of Ultrasound-Guided Water Enema for Secondary Intussusception in Children
}

\author{
Liezhen Hu, MD ${ }^{a}$, Bei Xia, MD ${ }^{a, *}$, Xiaopeng, Ma MD ${ }^{b}$, Tingting Liu, MD ${ }^{a}$, Tingting Ding, MD ${ }^{a}$, Dong Xiao, MD $^{b}$, \\ Bin Wang, MD ${ }^{b}$, Wei Yu, MD ${ }^{a}$, Zhou Lin, MD ${ }^{a}$, Lei Liu, MD ${ }^{a}$, Xia Feng, MD ${ }^{a}$ \\ ${ }^{a}$ Department of Ultrasonography, Shenzhen Children's Hospital, Shenzhen, Guangdong, China; ${ }^{b}$ Department of General Surgery, \\ Shenzhen Children's Hospital, Shenzhen, Guangdong, China \\ Received October 13, 2019; revision received November 17, 2019; accepted November 20, 2019
}

Objective: To investigate the diagnostic value of ultrasound-guided water enema for children with secondary intussusception.

Methods: We performed a retrospective analysis of the sonographic findings of 52 children with a confirmed diagnosis of SI through colonoscopy or surgical pathology between May 2016 and May 2019 in Shenzhen Children's Hospital. The diagnostic value of ultrasound-guided water enema was evaluated.

Results: Of the 52 patients with SI (29 male, 23 female; average age, 50 months [range: 3 months to 12 years]), 16 had intestinal polyps, including 15 cases of juvenile polyps and 1 case of Peutz-Jeghers (P-J) polyps; 14 had Meckel's diverticulum; 11 had lymphoma; 10 had intestinal duplication; and 1 had jejunal schwannoma. All 52 patients were diagnosed with intussusception, of which 41 were confirmed with the presence of pathological lead points (PLPs) before water enema. After enema, PLP was demonstrated in 48 cases. All of the remaining 4 cases without a PLP diagnosis were found to be Meckel's diverticulum. Ultrasonography revealed the specific features in each category. Patients with juvenile polyps showed hypoechoic masses in the intestinal lumen and some honeycomb-like anechoic masses while the single P-J polyp case showed hypoechoic masses with branching echoes in the intestinal lumen. Three of the Meckel's diverticula cases showed irregular hyperechoic masses inside the intussusception, and 7 showed cystic masses inside the intussusception. All 11 lymphomas cases showed extremely hypoechoic masses. All 10 intestinal duplication cases showed cystic masses at the neck or inside the intussusception, with walls exhibiting intestinal wall-like structure. The case of jejunal schwannoma showed irregular hypoechoic masses at the neck of the intussusception. There were no major complications such as intestinal perforation occurred in all the children after the completion of water enema ultrasound examination.

Conclusion: Ultrasound-guided water enema helps identify PLPs and improves the diagnosis of primary diseases in children with SI.

Key words: Ultrasound; Secondary intussusception; Water enema; Child

Advanced Ultrasound in Diagnosis and Therapy 2020;03:204-210

DOI: 10.37015/AUDT.2020.190029

I ntussusception, a condition in which a part of the intestine folds into the section immediately ahead of it, is one of the most common acute abdomen conditions affecting preschool-age children. It is is divided into two forms, primary intussusception and secondary intussusception, with secondary intussusception (SI) accounting for approximately $10 \%$ of all cases [1]. SI has pathological lead points (PLPs), which include polyps, Meckel's diverticulum, intestinal duplication, and lymphoma [2], but PLPs are only detected by ultrasound in about two-thirds of cases. Ultrasound-guided water enema facilitates the diagnosis of intestinal disorders and improves PLP identification. The current study retrospectively analyzed the ultrasonographic features and

* Corresponding author: Department of Ultrasonography, Shenzhen Children's Hospital, 7019, Yitian Road, Shenzhen, Guangdong, China

e-mail:xiabeimd@qq.com unrestricted use, distribution and reproduction in any medium provided that the original work is properly attributed. 
pathological results before and after water enema in 52 cases of SI and assessed the value of ultrasound-guided water enema in the diagnosis of SI.

\section{Materials and Methods}

\section{Subjects}

Fifty-two cases of children with SI were recruited from Shenzhen Children's Hospital between May 2016 and May 2019, including 29 males and 23 female patients. The average age of the subjects was 50 months ( 3 months $\sim 12$ years), with 4 between 0 and 6 months old; 13 between 6 months and 3 years old; 28 between 3 years and 7 years old; and 7 between 7 years and 12 years old. Clinical manifestations included 31 cases of abdominal pain, 23 cases of vomiting, 7 cases of bloating, 12 cases of hematochezia, 14 cases of abdominal mass, and 1 case of rectal prolapse. Among these cases of intussusception, 34 cases were the first occurrence, 8 cases were the second occurrence; 4 cases were the third; 5 cases were the fifth; and 1 case was the seventh (age distribution and recurrent frequency are shown in Table 1)

Table 1 Features of 52 recurrent intussusceptions secondary to PLPs

\begin{tabular}{lcc}
\hline Characteristics & Number & Proportion (\%) \\
\hline Age distribution & 4 & \\
$<6 \mathrm{~m}$ & 13 & 7.7 \\
$6 \mathrm{~m} \sim 3 \mathrm{y}$ & 28 & 25.0 \\
$3 \mathrm{~m} \sim 7 \mathrm{y}$ & 7 & 53.8 \\
$>7 \mathrm{y}$ & & 13.5 \\
\hline Recurrent frequency & 34 & \\
\hline 1 & 8 & 65.4 \\
2 & 4 & 15.4 \\
\hline 3 & 6 & 7.7 \\
\hline 3 & & 11.5 \\
\hline
\end{tabular}

All 52 cases were highly suspected of being SI. Patients with the following conditions were excluded from the current study: patients who showed poor general conditions with symptoms including shock and peritonitis; gastrointestinal perforation, as indicated by the presence of free gas in the abdominal cavity through $\mathrm{X}$-ray or color Doppler imaging; and intestinal necrosis on color Doppler imaging.

Family members of the patients signed the informed consent. This study was approved by the ethics committee of our hospital. All cases were confirmed by colonoscopy or surgical pathology.

\section{Apparatus and Methods}

\section{Apparatus}

All US examinations were performed with 4.5 9.0-MHz C2-9 convex array probes and 5.0 9.0$\mathrm{MHz} 9 \mathrm{~L}$ linear probes in the US system (LogiQ E9 Ultrasound System; GE Healthcare, Milwaukee, WI). And the mechanical index (MI) during the ultrasound examination was lower than 0.7 .

\section{Pre-enema preparation}

The following materials were used one infusion pole, one infusion tube, $500 \sim 1,000 \mathrm{~mL} 0.9 \%$ physiological saline at $37 \sim 40{ }^{\circ} \mathrm{C}$, one catheter, one $50 \mathrm{~mL}$ syringe, a pair of hemostatic forceps, and a pack of liquid paraffin.

\section{Procedure}

All tubes were confirmed as leak-proof. The infusion tube was connected to the catheter. The catheter was inserted into the anus to a depth of $7 \sim 10 \mathrm{~cm}$. Saline infusion was started after the air sac was filled with air using the $50-\mathrm{mL}$ syringe and fixed. The catheter was removed after examination.

We monitored liquid passage through the rectum, sigmoid, descending colon, transverse colon, ascending colon, ileocecal valve and ileum. We examined the presence of space-occupying lesions such as polyps in the intestinal lumen, whether there were abnormal echoes or thickening of the intestinal wall, mass locations (intraintestinal or extraintestinal). or lesions at the neck or inside the intussusception.

\section{Results}

The lengths of the intussusception ranged from 3.0 $\sim 18.0 \mathrm{~cm}$, and the diameters of the outer rims ranged from $2.5 \sim 4.5 \mathrm{~cm}$. Peritoneal effusion was found in 10 cases. Effusion was found in the sleeve of the intussusception in 5 cases. All cases were diagnosed with intussusception by color Doppler ultrasonography prior to water enema, with the long axis showing the "sleeve sign" and the short axis showing the "target sign". The ultrasonographic features and pathological results obtained for patients with different PLPs were as follow:

Intussusception secondary to polyps: Juvenile polyps were found in 13 cases, with 12 cases showing hypoechoic masses at the neck or inside the intussusception in the ultrasonography before water enema. Masses were localized at the neck or inside the intussusception within the intestinal lumen. The echoes were irregular within the masses, with some honeycomb-like anechoic masses. Color Doppler flow imaging (CDFI) revealed rich, branching blood flow signals (Fig. 1). After water enema, polyps were 
found at the descending colon in 7 cases, the ascending colon in 2 cases, and the ileocecal junction in 3 cases. The sizes of the largest and smallest polyps were $4.0 \times 2.0 \mathrm{~cm}$ and $1.7 \times 1.5 \mathrm{~cm}$, respectively. The polyps contained pedicles that were attached to the intestine wall. Consistent results were obtained by water enema and colonoscopy. All patients underwent colonoscopy and polypectomy. Juvenile polyps were confirmed by pathological analysis after polypectomy. One case of a missed diagnosis of a rectal polyp was identified with a size of $3.0 \times 2.5 \mathrm{~cm}$ after water enema. Diagnosis was confirmed after colonoscopy and polypectomy. One case was diagnosed with Peutz-Jeghers (P-J) syndrome. The patient had a dark blotch at the lower lip, and intussusception was found at the upper abdomen with a length of approximately $18 \mathrm{~cm}$ and an outer diameter of $4.5 \mathrm{~cm}$. There was a hypoechoic mass of $2.8 \times 2.0 \mathrm{~cm}$ at the neck of the intussusception, showing branching echoes. CDFI revealed dot-like and stripe-like signals of blood flow within the hypoechoic mass (Fig. 2). The length of the intussusception did not shorten when fluid
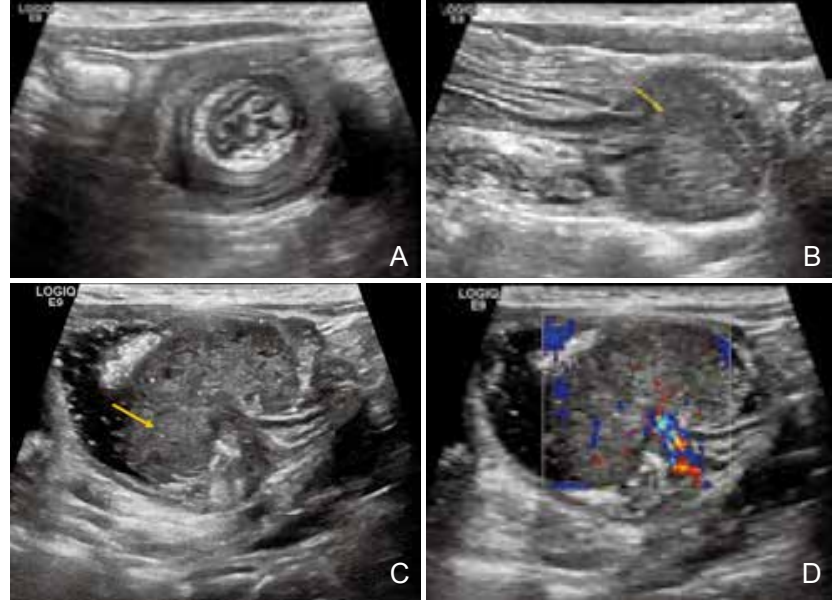

Figure 1 The ultrasonic examination of a 23-month-old girl showing SI to polyp which was confirmed through colonoscopy, and the intussusception was found in the left lower quadrant. (A) The short axis showing the "target sign"; (B) The long axis showing the "sleeve sign", the neck of the intussusception showing hypoechoic masses with some honeycomb-like anechoic masses (arrow) in the ultrasonography; (C) Polyps (arrow) were found at the descending colon after water enema, and the polyps contained pedicles that were attached to the intestine wall; (D) CDFI shows the rich, branching blood flow signals.
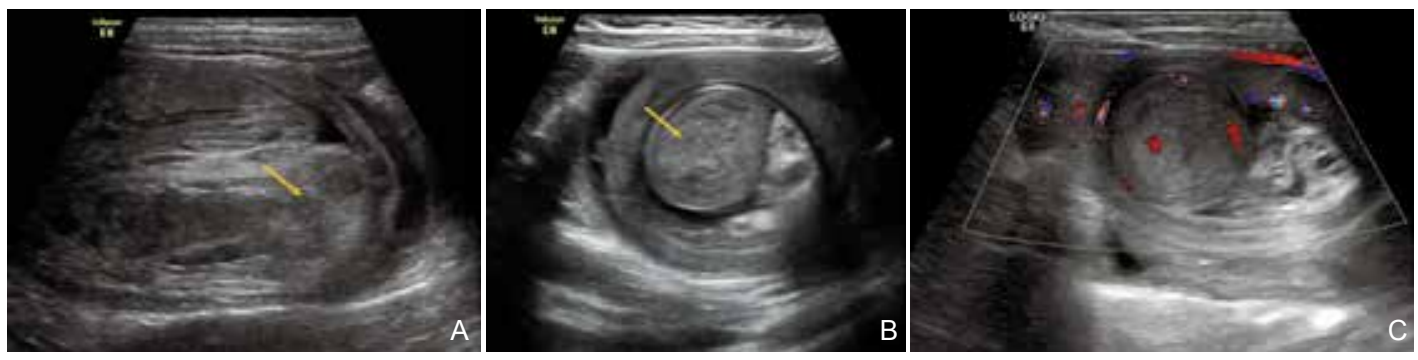

Figure 2 The ultrasonic examination of a boy (age: 7 years, 5 months) showing P-J syndrome-induced SI which was confirmed by laparotomy. Ultrasound shows the features of intussusception in the upper abdomen. (A) The long axis showing the "sleeve sign", the neck of the intussusceptions showing a polyp (arrow) with hypoechoic masses, and the echoes were irregular within the masses in the ultrasonography; (B) The short axis showing the "target sign", the neck of the intussusception (arrow) showing a polyp (arrow); (C) CDFI revealed dot-like and stripe-like signals of blood flow within the hypoechoic mass.

passed through ileocecal valve and reached the colon after water enema. Therefore, it was determined to be a polyp-induced SI of the small intestine. Laparotomy confirmed that the intussusception was secondary to P-J syndrome (three polyps of the small intestine, with the largest at $2.5 \mathrm{~cm}$ and the smallest at $0.5 \mathrm{~cm}$ ). A portion of the intestine was dark red, indicating ischemia.

Intussusception secondary to Meckel's diverticulum: There were 14 cases of Meckel's diverticulum, of which 5 cases were diagnosed as primary disease prior to water enema. Ultrasonography revealed a cystic mass at the neck of the intussusception, the sizes of the largest and smallest mass were $4.0 \times 2.0 \mathrm{~cm}$ and $1.5 \times 1.2 \mathrm{~cm}$, along with a thickened mucous layer and intensified echo. CDFI revealed dot-like blood flow signals at the wall of the cystic mass (Fig. 3). Primary disease was identified in 5 more cases -3 cases showing irregular hyperechoic masses inside the intussusception, all of which were folded diverticulum echoic masses (Fig. 4), and 2 cases showing cystic masses inside the intussusception. The primary disease was not identified in five cases after water enema. Surgery confirmed Meckel's diverticuluminduced SI in all 14 cases.

Intussusception secondary to intestinal duplication: All 10 cases were diagnosed with primary disease prior to enema. Ultrasonography revealed cystic masses at the neck of the intussusception with walls exhibiting an intestinal wall-like structure. The size of the largest mass was $3.8 \times 2.3 \mathrm{~cm}$, and the smallest was $2.4 \times 1.4 \mathrm{~cm}$. After water enema, the cystic mass was found outside the intestinal lumen, with no obvious change in size or morphology. CDFI revealed dot-like and stripe-like blood flow signals at the wall of the cystic mass (Fig. 5). Six cases were confirmed with ileum duplication, and 4 cases were confirmed with ileocecal duplication by surgery. 
Intussusception secondary to lymphoma: Ten cases were diagnosed with intussusception secondary to lymphoma prior to enema. Ultrasonography showed extremely hypoechoic masses at the neck of the intussusception with evenly distributed echoes. The sizes of the largest and smallest lymphoma were $3.0 \times 1.5 \mathrm{~cm}$ and $2.0 \times 1.2 \mathrm{~cm}$, respectively. The lymphomas were

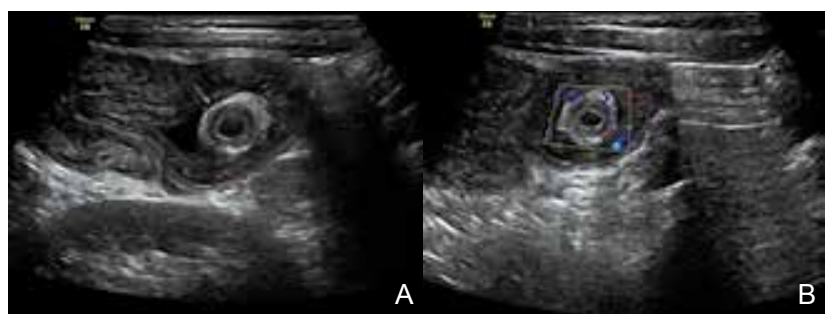

Figure 3 The ultrasonic examination of a boy (age: 3 years, 4 months) showing intussusception secondary to Meckel's diverticulum which was confirmed by surgery, and the intussusception was found in the right upper abdomen. (A) The long axis showing the "sleeve sign", the neck of the intussusception showing a Meckel's diverticulum (arrow) revealed a cystic mass along with a thickened mucous layer and intensified echo in the Ultrasonography; (B) CDFI revealed dot-like blood flow signals at the wall of the cystic mass.

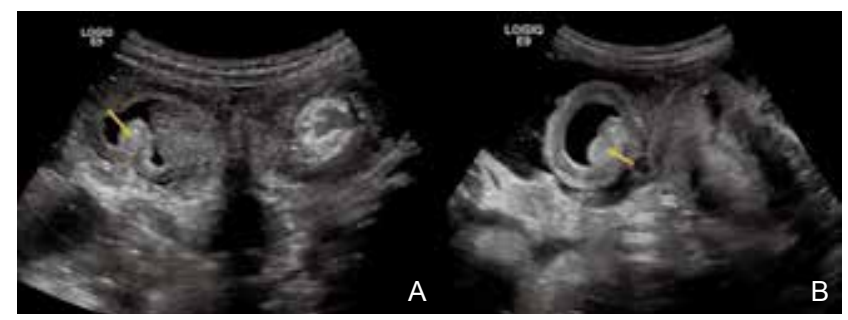

Figure 4 The ultrasonic examination of a boy (age: 1 year, 4 months) showing intussusception secondary to Meckel's diverticulum which was confirmed by surgery. (A) The short axis showing the "target sign", the tortuous sleeve of the intussusception showing a Meckel's diverticulum (arrow) with hyperechoic masses; (B) It easier to expose Meckel's diverticulum(arrow) after water enema.

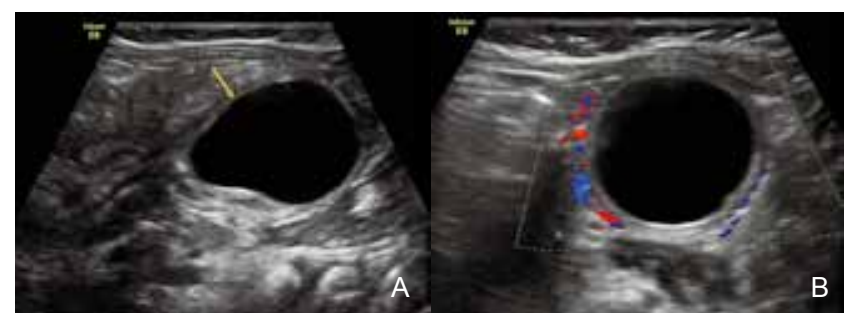

Figure 5 The ultrasonic examination of a girl (age: 3 months) showing intussusception secondary to intestinal duplication which was confirmed by surgery. (A) The long axis showing the "sleeve sign". Ultrasonography revealed cystic masses (arrow) at the neck of the intussusception with walls showing an intestinal wall-like structure; (B) CDFI revealed dot-like blood flow signals at the wall of the cystic mass.

located at the ileocecum in 6 cases and at the end of the ileum in 4 cases. CDFI showed ample branched blood flow signals (Fig. 6). In one case of a missed diagnosis before enema, intussusception occurred 5 times. The primary disease was not diagnosed the first 4 times. Lymphoma was found in the ileum after enema, $3.0 \mathrm{~cm}$ away from the ileocecum, with a size of $2.0 \times 1.2 \mathrm{~cm}$.

Other SI: Intussusception was secondary to jejunum schwannoma in the remaining case. Ultrasonography showed an irregular hypoechoic mass at the neck of the intussusception, revealing no clear boundary with the intestinal wall. It was difficult to distinguish this mass

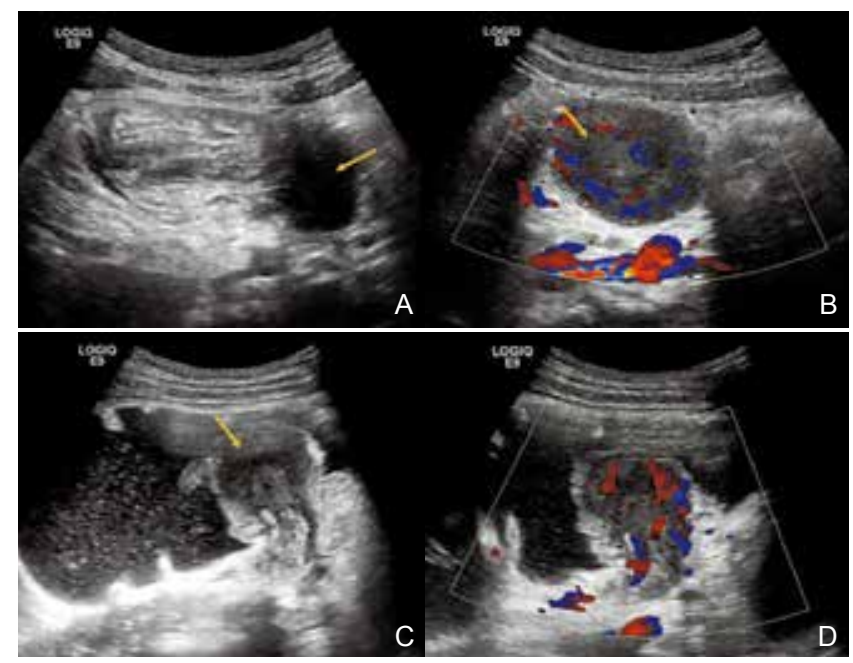

Figure 6 The ultrasonic examination of a boy (age: 5 years, 10 months) boy showing intussusception secondary to Burkitt lymphoma localized at the ileocecum, which was confirmed by surgery. (A) The long axis showing the "sleeve sign", the neck of the intussusception showing lymphoma with hypoechoic masses (arrow); (B) CDFI revealed ample blood flow signals within lymphoma (arrow); (C) Lymphoma is located at the ileocecum after water enema, with masses (arrow) protruding into the intestinal lumen; (D) CDFI revealed ample blood flow signals within lymphoma.

and the thickened intestinal wall. CDFI revealed no obvious blood flow signal. After the fluid passed through the ileocecal valve to the colon following water enema, no shortening of the intussusception was noticed, and it was considered a small intestinal intussusception. Surgery confirmed that the intussusception was secondary to jejunum schwannoma.

\section{Discussion}

Intussusception refers to the insertion of part of the intestine into an adjacent part of the intestine, leading to mechanical intestinal obstruction. There are two forms of intussusception: primary and secondary. Primary intussusception usually occurs in children between 5 months and 3 years of age, while SI usually occurs in children older than 3 years of age. One study reported that SI occurs in approximately 1 in 10 cases of SI [1], while an analysis of 11,025 cases of intussusception by Lin et al. in China indicated that SI accounts for approximately $6 \%$ of all intussusceptions [3]. Blakelock et al. reported that approximately $60 \%$ of SI occurs in children between 5 and 14 years of age, with an increasing incidence with age. SI relapses more 
frequently compared with primary intussusception, with a recurrence rate ranging from $2 \%$ to $20 \%$ [4]. Most primary intussusceptions $(89 \%)$ are located at the right upper quadrant since the major forms are ileocolic and ileocecal. In contrast, the position of the SI is usually uncertain and may be ileocolic, ileoileal, colocolic, or another type [4,5]. In the current study, SI in the right upper quadrant was observed in 10 cases, accounting for $47 \%$ of all cases. Intestinal wall edema, ischemia, and necrosis occur more frequently in patients with SI than does primary intussusception due to the pulling of the intestine by PLP, the insertion of mesenteric tissue and lymph nodes into the intestine, and the pressing of the mesenteric vessels of the intestinal wall by PLP. If it is not remitted, SI more easily leads to fluid accumulation in the sleeve of the intussusception or the abdominal cavity and intestinal obstruction, which frequently occurs in older children. Among the 37 cases of SI reported by Zhang et al., SI showed longer and thicker intussusception compared with primary intussusception in the control group [6]. One characteristic of SI is its high tendency to relapse due to the presence of PLP, and Navarro et al. suggested that SI can be predicted by the recurrence of intussusception [7]. Consistently, PLPs were not identified in 11 cases prior to enema in our study; however, they were identified in 7 cases when enema was performed due to multi-recurrences and larger outer diameter of the intussusception.

Recently, ultrasound has become the primary technique for the diagnosis of intussusception. The typical sonography shows a "sleeve sign" on the long axis and "target sign" on the short axis, and the sonographies in all cases in the current study exhibited this pattern. Ultrasound has a high specificity and sensitivity for the diagnosis of intussusception, with some reports showing $100 \%$ specificity and sensitivity [8]. In addition to the diagnostic characterization of intussusception, ultrasound can identify PLPs in some SI cases. According to studies by Danneman et al. two-thirds of the SI cases were accurately diagnosed by ultrasound [2,9]. In these studies, water enema was not frequently used in assisting ultrasound-guided examination. Water enema was used to assist in the diagnosis of intestinal diseases as early as 1990. Filling the intestine with fluid detaches the anterior and posterior intestinal walls and disperses the interfering gas inside the intestine, leading to a clearer display of the intestinal wall structure, echoes, and lesions in the intestinal lumen. Therefore, water enema ideally assists imaging and, thus, the diagnosis of multiple intestinal diseases, such as Crohn's disease, intestinal polyps, intestinal tuberculosis, infective enteritis, intestinal ischemia and intestinal lymphoma [10]. The most prevalent use of ultrasound-guided water enema is the diagnosis of colon polyps. Since Walter et al. first reported the use of ultrasound-guided water enema in the diagnosis of juvenile polyps in 1992, an increasing number of authors have utilized ultrasound-guided water enema in the diagnosis of colon polyps [11]. However, few studies have reported the use of ultrasound-guided water enema in assisting in the diagnosis of PLP of other types of SI in addition to colon polyps. Our study utilized ultrasound-guided water enema to assist the diagnosis of primary diseases of SI. Among the 52 SI patients, PLP was identified in 41 cases prior to water enema, with a diagnosis rate of $78.8 \%$, and a diagnosis was made in 48 cases after water enema, with a diagnosis rate of $92.3 \%$. Thus, water enema substantially improved the diagnosis of PLP by ultrasound compared with the diagnosis prior to water enema.

The ultrasonographic features of PLPs and the diagnostic value of ultrasound-guided water enema for different types of SI are summarized below.

Polyps: Juvenile polyps usually occur in children younger than 10 years of age, with the polyps commonly found in the rectum and sigmoid. The polyps are benign hamartomas, with normally one polyp detected in each patient. However, if more than 5 juvenile polyps are present simultaneously, there is a tendency to become malignant [12]. Following the injection of physiological saline through the anal tube, fluid sequentially fills the rectum, colon, and ileum. Fluid disperses the interfering gas inside the intestine, making it easier to expose and recognize polyps in the intestine at the presence of the anechoic fluid. Ultrasonography of juvenile polyps shows hypoechoic masses in the intestinal lumen with pedicles attached to the intestinal wall, accompanied by honeycomb-like anechoic areas. CDFI shows rich branching blood flow signals $[10,11,12]$. The sonographic features of juvenile polyps in the current study were consistent with previous studies. Colon polyps are easily identified by ultrasound-guided water enema, especially in the presence of intussusception. Matteo noted that the diagnosis of polyps in the flexura hepatica coli can be easily missed. Another factor leading to a missed diagnosis is the small size of polyps. The rectum is located at the deep side of the pelvis, so the probe cannot press the rectum effectively. Similarly, it is not easy to acquire a complete scan of flexura hepatica coli, leading to a missed diagnosis, especially of small polyps [11]. In the current study, there was one case of a missed diagnosis of a rectal polyp in two examinations prior to water enema, and the diagnosis was confirmed after water enema. Rectal prolapse is the initial clinical symptom in some cases of intussusception secondary to colon polyps. In the current study, we found one case of rectal prolapse. Thus, it is important to determine 
whether patients have intussusception secondary to rectal or colon polyps if rectal prolapse is their initial symptom. The Peutz-Jeghers polyp occurs most frequently in the small intestine, with a sequence of jejunum, ileum and duodenum, followed by the colon and stomach. Sonography usually shows hypoechoic or median echoic masses accompanied by branching hypoechoes, of which lobulated masses are more common, with a pedicleshaped structure surrounding the polyp. The pathological mechanism may involve the insertion of smooth muscle tissue in a branch-like pattern that is slightly hypoechoic. CDFI shows rich branching blood flow signals. Small bowel intussusception is the most common form of intussusception secondary to the Peutz-Jegher polyp, and it is frequently accompanied by severe complications, such as bowel obstruction, ischemia and necrosis $[13,14]$. The current study includes one case of P-J syndrome, with multiple (three) polyps found in the small intestine. During surgery, the intestine was found to be dark red, indicative of ischemia, consistent with previous reports. Small intestine intussusception is the most common form of intussusception secondary to the Peutz-Jegher polyp. However, if it is located in the right abdomen, it is difficult to distinguish it from ileocolic intussusception. Small intestine intussusception is determined by the failure of fluid to reach the intussusception once it passes through the ileocecal valve and the absence of shortening of the mass after water enema. Water enema provides a direction regarding further therapeutic treatment.

Meckel's diverticulum: Meckel's diverticulum is a common congenital anomaly of the small intestine that occurs due to an incompletely obliterated embryonic omphalomesenteric duct attached to the distal end of the small intestine, with an incidence of approximately $2 \%[15,16]$. Meckel's diverticulum is the most common cause of SI. The clinical features of Meckel's diverticulum-induced SI are that it is not easy to perform air or water enema and that remission frequently occurs after a successful enema [3,6]. It is difficult to diagnose Meckel's diverticulum in the absence of inflammation and effusion, and the occurrence of intussusception secondary to Meckel's diverticulum also makes it difficult to diagnose [6,9]. Due to the connection between the diverticulum and the ileum, it is extremely difficult to distinguish Meckel's diverticulum from normal intestine in the absence of either inflammation or effusion. The amorphous features of Meckel's diverticulum make it more difficult to identify than intestinal duplication. Among the 14 cases in our current study, only 5 cases were diagnosed prior to water enema, and 4 cases were missed diagnoses even after water enema. Fluid disperses the interfering gas inside the intestine, making it easier to expose PLPs in the presence of anechoic fluid after water enema. The sonographic features of the 10 cases of Meckel's diverticulum-induced SI were as follows. Seven cases showed a cystic mass at the neck of the intussusception with a thick mass wall that lacked a clear boundary between the intestine, along with a thickened mucous layer and intensified echo. Three cases showed irregular hyperechoic masses at the neck of the intussusception, which were folded diverticulum echoic masses. The hyper echoes resulted from reflections from multi-layered interfaces. The pathological mechanisms of this phenomenon included the reflections from the multi-layered mucosa interfaces caused by the ectopic gastric or pancreatic mucosa of the diverticulum and inflammation. The sonographic features of Meckel's diverticulum in our study were consistent with those shown by Zhang et al. [6].

Intestinal duplication: Intestinal duplication refers to the congenital duplication of a structure that is similar to the intestinal wall structure. It may occur anywhere along the gastrointestinal tract, most commonly in the ileum. Intestinal duplication is divided into the intraintestinal cystic type, extraintestinal cystic type and tubular type, according to the pathological basis. It is cystic or tubular in shape according to sonographic features, with the extraintestinal cystic type occurring in more than $80 \%$ of duplication masses $[17,18]$. Ultrasonography revealed cystic masses at the neck of the intussusception with walls showing an intestinal wall-like structure in all 10 cases in the current study. CDFI revealed dotlike blood flow signals at the wall of the cystic mass. A definite diagnosis could be made of SI induced by intestinal duplication. Water enema helped identify the localization of the intestinal duplication, distinguishing it from Meckel's diverticulum and determining whether the cystic mass was inside or outside the intestinal lumen.

Intestinal tumors: Water enema has high diagnostic value in the identification of neoplastic diseases of the intestinal wall. Lymphoma is the most common intestinal tumor, and the incidence of other malignant tumors is very low [19]. The current study included 11 cases of lymphoma and 1 case of jejunum schwannoma. The lymphoma that induces intussusception is mainly the nodule type. Lymphomas that lead to diffuse thickening of the intestinal wall or that form large masses do not normally induce intussusception. The position of the tumor, particularly lymphoma localized at the ileum or ileocecum, can be determined by water enema, which shows the relationship between the masses and the intestinal wall. Ultrasonography showed extremely hypoechoic and regularly shaped masses at the neck of the intussusception, with evenly distributed echoes in four cases of lymphoma. CDFI showed ample branched blood flow signals. No clear boundary was identified 
between the masses and the intestinal wall after water enema, with masses protruding into the intestinal lumen. Sonography of lymphoma has specific features that allow the diagnosis of some lymphomas based on sonography. However, most intestinal tumors require confirmation by surgical pathology $[6,19]$.

In summary, ultrasound-guided water enema is not only a safe and effective way to diagnose various intestinal diseases $[10,11,12]$, but it is also of substantial clinical value in the detection of PLPs in patients with SI. It not only assists in the detection of thickened lesions and neoplastic diseases of the intestine wall but also has high value in distinguishing masses inside and outside the intestine. Ultrasound-guided water enema substantially increases the diagnosis rate of SI, provides direction for its treatment and can be used as a routine examination for suspected SI.

\section{Conflicts of Interest}

The authors declare that they have no conflicts of interest.

\section{References}

[1] Marsicovetere P, Ivatury SJ, White B, Holubar SD. Intestinal intussusception: etiology, diagnosis, and treatment. Clin Colon Rectal Surg 2017;30:30-39.

[2] Daneman A, Alton DJ, Lobo E, Gravett J, Kim P, Ein SH. Patterns of recurrence of intussusception in children: a 17-year review. Pediatr Radiol 1998;28:913-919.

[3] Lin XK, Xia QZ, Huang XZ, Han YJ, He GR, Zheng N. Clinical characteristics of intussusception secondary to pathologic lead points in children: a single-center experience with 65 cases. Pediatr Surg Int 2017;33:793-797.

[4] Blakelock RT, Beasley SW. The clinical implications of nonidiopathic intussusception. Pediatr Surg Int 1998;14:163-167.

[5] Edwards EA, Pigg N, Courtier J, Zapala MA, MacKenzie JD, Phelps AS. Intussusception: past, present and future. Pediatr Radiol 2017;47:1101-1108.

[6] Zhang Y, Dong Q, Li SX, Ren WD, Shi B, Bai YZ, et al. Clinical and ultrasonographic features of secondary intussusception in children Eur Radiol 2016;26:4329-4338.

[7] Navarro OM, Daneman A, Chae A. Intussusception: the use of delayed, repeated reduction attempts and the management of intussusceptions due to pathologic lead points in pediatric patients. AJR Am J Roentgenol 2004;182:1169-1176.

[8] Wiersma F, Allema JH, Holscher HC. Ileoileal intussusception in children: ultrasonographic differentiation from ileocolic intussusception. Pediatr Radiol 2006;36(11):1177-1181.

[9] Ntoulia A, Tharakan SJ, Reid JR, Mahboubi S. Failed Intussusception Reduction in Children: Correlation Between Radiologic, Surgical, and Pathologic Findings. AJR Am J Roentgenol 2016;207:424-433.

[10] Maconi G, Radice E, Bareggi E, Porro GB. Hydrosonography of the gastrointestinal tract. AJR Am J Roentgenol 2009;193:700-708.

[11] Baldisserotto M, Spolidoro JV, Bahú Mda G. Graded compression sonography of the colon in the diagnosis of polyps in pediatric patients. AJR Am J Roentgenol 2002;179:201-205.

[12] Takahashi T, Miyano G, Kayano H, Lane GJ, Arakawa A, Yamataka A. A child with colo-colonic intussusception due to a large colonic polyp: Case report and literature review. Afr J Paediatr Surg 2014;11:261-263.

[13] Singh A, Vidyarthi SH, Kasliwal N, Khandelwal S, Sharma VK Triple site intussusceptions in Peutz-Jeghers syndrome. ANZ J Surg 2019;89:E153-E155.

[14] Wu L, Iyer RS, Drugas GT, Stanescu AL. Unusual ultrasound appearance of small bowel intussusception and secondary bowel obstruction in a child with Peutz-Jeghers syndrome. Clin Imaging 2017;43:136-139.

[15] Menezes M, Tareen F, Saeed A, Khan N, Puri P. Symptomatic Meckel's diverticulum in children: a 16-year review. Pediatr Surg Int 2008;24:575-577.

[16] Baldisserotto M, Maffazzoni DR, Dora MD. Sonographic findings of Meckel's diverticulitis in children. AJR Am J Roentgenol 2003;180:425-428.

[17] Zouari M, Bouthour H, Abdallah RB, Hlel Y, Malek RB, Gharbi Y, et al. Alimentary tract duplications in children: report of 16 years' experience. Afr J Paediatr Surg 2014;11:330-333.

[18] Di Serafino M, Mercogliano C, Vallone G. Ultrasound evaluation of the enteric duplication cyst: the gut signature. $J$ Ultrasound 2015;19:131-133.

[19] Ghimire P, Wu GY, Zhu L. Primary gastrointestinal lymphoma. World J Gastroenterol 2011;17:697-707. 